\title{
Incidencia de lesión de vías biliares en un hospital universitario: análisis de más de 1.600 colecistectomías laparoscópicas
}

\author{
Incidence of bile duct lesion at a university hospital: analysis of more than \\ 1,600 laparoscopic cholecystectomies
}

\author{
Juan Carlos Serna1', Sara Patiño², Melissa Buriticá², Érika Osorio², Carlos Hernando Morales ${ }^{3}$, \\ Juan Pablo Toro ${ }^{4}$ \\ 1 Médico, residente de Cirugía General, Universidad de Antioquia, Medellín, Colombia \\ 2 Estudiante de Medicina, Universidad de Antioquia, Medellín, Colombia \\ 3 Médico, cirujano general, M. Sc. en Epidemiología Clínica; profesor titular de Cirugía General, Universidad de Antioquia; cirujano \\ asistencial, Hospital Universitario San Vicente Fundación, Medellín, Colombia \\ 4 Médico, cirujano general, especialista en Cirugía Mínimamente Invasiva; profesor asistente de Cirugía General, Universidad de \\ Antioquia; cirujano asistencial, Hospital Universitario San Vicente Fundación, Medellín, Colombia
}

Trabajo presentado en el Simposio Nacional del Residente Quirúrgico, XLIV Congreso Nacional "Avances en Cirugía", Cartagena, Colombia, agosto de 2018.

\section{Resumen}

Introducción. La colecistectomía laparoscópica es el procedimiento laparoscópico más comúnmente realizado por el cirujano general. La lesión de la vía biliar es la complicación más temida debido a sus implicaciones clínicas y económicas. El objetivo de esta investigación fue determinar la incidencia de la lesión de la vía biliar en un centro de formación académica e identificar los posibles factores de riesgo asociados a su presentación.

Materiales y métodos. Se llevó a cabo un estudio observacional retrospectivo en pacientes a quienes se les practicó una colecistectomía laparoscópica en el Hospital Universitario San Vicente Fundación de Medellín entre marzo de 20II y septiembre 20I6. Se realizó un análisis univariado y bivariado para explorar la asociación de algunas variables preoperatorias e intraoperatorias con la lesión de la vía biliar. Se utilizó el programa estadístico Stata $2014^{\mathrm{TM}}$.

Resultados. Se incluyeron I.6or pacientes. La incidencia de lesión de la vía biliar fue de o,8 \% (I4 pacientes), con una mortalidad global de o,4 \%. La mayoría de estas lesiones fueron tipo A de Strasberg; solo dos pacientes requirieron una reconstrucción compleja de la vía biliar. Se encontró relación estadísticamente significativa con mayor tiempo operatorio $(\mathrm{p}<0,05)$ y mayor sangrado intraoperatorio $(\mathrm{p}<0,05)$ con la presentación de lesión de la vía biliar. Conclusión. La colecistectomía laparoscópica es un procedimiento seguro en el Hospital Universitario San Vicente Fundación, centro de formación de residentes quirúrgicos. La tasa de lesión de la vía biliar es similar a la reportada en la literatura.

Palabras clave: conducto colédoco; colelitiasis; colecistitis aguda; colecistectomía laparoscópica; complicaciones intraoperatorias.

Fecha de recibido: 8/06/2018. Fecha aceptación: 28/08/2018.

Correspondencia: Juan Carlos Serna, MD, Calle 51 \# 45-93 Consultorio 201, Clinica Soma, Teléfono: 5121749, Medellín, Colombia Correo electrónico. juankar2687@hotmail.com

Citar como: Serna JC, Patiño S, Buriticá M, Osorio É, Morales CH, Toro JP. Incidencia de lesión de vías biliares en un hospital universitario: análisis de más de 1.600 colecistectomías laparoscópicas. Rev Colomb Cir. 2019;34:45-54. https://doi.org/10.30944/20117582.97

Este es un artículo de acceso abierto bajo una Licencia Creative Commons - BY-NC-ND https://creativecommons.org/licenses/by-nc-nd/4.0/deed.es 


\begin{abstract}
Introduction. Laparoscopic cholecystectomy is the most common laparoscopic procedure performed by the general surgeon. Bile duct injury (BDI) is the most feared complication due to its clinical and economic implications. The goal of this study was to determine the incidence of BDI at a teaching hospital and to identify possible related risk factors.
\end{abstract}

Methods. This is an observational retrospective study that included patients who underwent laparoscopic cholecystectomy at San Vicente Foundation University Hospital (HUSVF) in Medellin, Colombia, in the period 20II to September 20I6. Univariate and bivariate analyses were performed to explore possible association of some perioperative variables with BDI. Statistic software STATA 2014 was used.

Results. A total of I,60I patients were included. BDI incidence was $0.8 \%$ (I4 patients), global mortality was $0.4 \%$. Most of BDIs were type A of the Strasberg classification and only two patients required complex reconstructions of the bile ducts. Longer operative time ( $p>0.05)$ and higher intraoperative blood loss ( $p<0.05)$ were related with BDI.

Conclusion. Laparoscopic cholecystectomy is a safe procedure at HUSVF, a training center for surgical residents. Bile duct injury rate at this hospital is similar to that reported in the literature.

Key words: common bile duct; cholelithiasis; cholecystitis, acute; cholecystectomy, laparoscopic; intraoperative complications.

\section{Introducción}

En Colombia, se practican anualmente, aproximadamente, 60.000 colecistectomías laparoscópicas, urgentes o electivas, lo cual la hace el procedimiento laparoscópico más frecuente ${ }^{\mathrm{I}}$. A pesar de que la colecistectomía laparoscópica es muy segura, la lesión de las vías biliares es la complicación más temida por sus implicaciones clínicas y económicas, además de su potencial asociación con otras complicaciones, como las lesiones vasculares y viscerales.

Desde los comienzos de la globalización de la técnica, se ha hecho énfasis en esta problemática $y$, en consecuencia, se han ideado estrategias que buscan facilitarle al cirujano una buena identificación y reconocimiento de las estructuras anatómicas clave. Estas estrategias procuran que el procedimiento sea más seguro y que cuando no sea posible hacer una disección segura, se opte por alternativas y variaciones de la técnica que permitan evitar una complicación mayor ${ }^{2-4}$.

En los Estados Unidos, la lesión de las vías biliares es la causa más común de demandas penales durante cirugía gastrointestinal, y le corresponden el $50 \%$ de las demandas por cirugía laparoscópica ${ }^{5,6}$.
En el Hospital Universitario San Vicente Fundación, la colecistectomía por laparoscopia es un procedimiento usualmente practicado por un residente de Cirugía General, con el acompañamiento y la supervisión del cirujano general asistencial. Sin embargo, no existen estudios que muestren cuál es la incidencia real de lesiones de las vías biliares en este hospital, que permitan compararla con lo reportado a nivel mundial y que, asimismo, permitan evaluar la seguridad del procedimiento en los hospitales donde se forman profesionales de la salud.

El objetivo de esta investigación fue determinar la incidencia de las lesiones de las vías biliares en el Hospital Universitario San Vicente Fundación e identificar los posibles factores de riesgo asociados a su presentación.

\section{Materiales y métodos}

Se llevó a cabo un estudio descriptivo de tipo retrospectivo en el Hospital Universitario San Vicente Fundación, desde marzo de 20II hasta diciembre de 20I6, utilizando como criterio de inclusión ser un paciente de 15 o más años de edad sometido a colecistectomía laparoscópica en la institución. 
Se excluyeron los pacientes menores de I5 años, aquellos a quienes se les había practicado colecistectomía en otra institución, los remitidos de otras instituciones con lesión de la vía biliar y aquellos con lesión de la vía biliar por colecistectomía abierta.

Los resultados primarios fueron la incidencia de lesión de las vías biliares y la identificación de posibles factores de riesgo para su presentación.

Se obtuvo el aval de los comités de investigaciones y de ética médica de la Universidad de Antioquia y del Hospital Universitario San Vicente Fundación para la realización del proyecto.

Se analizaron algunas variables demográficas y condiciones clínicas antes, durante y después de la cirugía. Se hizo un análisis descriptivo univariado para las variables cuantitativas con medias y desviación estándar (distribución normal) o medianas y rango intercuartílico (RIQ) (distribución no normal), y para las variables categóricas, en porcentajes. Posteriormente, se hizo un análisis bivariado de independencia (tablas de contingencia) por medio de la prueba de ji al cuadrado o la prueba exacta de Fisher para las variables categóricas y, para las variables continuas, el test de Mann-Whitney, con un valor p de significación estadística menor de 0,05. Se utilizó el programa estadístico Stata ${ }^{\mathrm{TM}}$, versión $2 \mathrm{OI} 4$.

\section{Resultados}

\section{Población}

Desde marzo de 20II hasta diciembre 20I6, se incluyeron I.6or pacientes a quienes se les practicó colecistectomía laparoscópica. En la población estudiada (tabla I), la mayoría fueron mujeres (76,5\%), las edades fluctuaron entre los I5 y los 99 años, la edad promedio fue de 46 años, y el grupo etario predominante (4I \%) fue el de I5 a 40 años de edad.

La tasa de conversión a cirugía abierta fue de o,82 \% (I3 pacientes) y la mayoría de los pacientes analizados no tenían historia previa de colelitiasis (1396; 87,2 \%). En 492 (30,7 \% de la muestra), se reportó historia previa de cirugía abdominopélvica, la mayoría correspondiente a cirugías
Tabla 1. Características de los pacientes llevados a colecistectomía laparoscópica

\begin{tabular}{lcc}
\hline Variable & Total & $\begin{array}{c}\text { Porcentajel } \\
\text { Rango }\end{array}$ \\
\hline Género Femenino & 1225 & $76.5 \%$ \\
Edad promedio años ( $\mathrm{n}=1598)$ & 46 & $15-99$ \\
Diabetes & 136 & $8.5 \%$ \\
Obesidad & 87 & $6.8 \%$ \\
Cirugía Previa & 492 & $30.7 \%$ \\
Cirrosis & 6 & $0.4 \%$ \\
\hline Tiempo de diagnóstico de colelitiasis en años $(\mathrm{n}=1560)$ \\
\hline$\leq 2$ años & 1528 & $98 \%$ \\
$>2$ hasta 5 años & 21 & $1.4 \%$ \\
$>5$ hasta 10 años & 7 & $0.5 \%$ \\
$>10$ años & 4 & $0.3 \%$ \\
\hline Tiempo de evolución de colecistitis aguda en días $(\mathrm{n}=1444)$ \\
\hline$\leq 3$ días & 1126 & $78 \%$ \\
$>3$ hasta 7 días & 249 & $17.2 \%$ \\
$>7$ días & 69 & $4.8 \%$ \\
Cirugía urgente & 1437 & $89.8 \%$ \\
Colecistectomía total & 1595 & $99.6 \%$ \\
Colecistectomía subtotal & 5 & $0.3 \%$ \\
Colecistostomía & 2 & $0.1 \%$ \\
Tiempo quirúrgico promedio & 61 & $18-555$ \\
minutos & & \\
Sangrado promedio mL ( $\mathrm{n}=1595)$ & 15 & $5-1100$ \\
Estancia hospitalaria promedio & 1 & $1-56$ \\
días ( $\mathrm{n}=1089$ ) & & \\
\hline & &
\end{tabular}

ginecológicas (cesárea, histerectomía, ligadura de trompas, etc.). Hubo un número relativamente bajo de pacientes en quienes se reportó obesidad como enfermedad concomitante $(5,45 \%)$.

El 89,78 \% de las colecistectomías fueron urgentes y, en el $70 \%$ de los casos de colecistitis aguda, la cirugía se practicó en 72 horas o menos después del ingreso. Los cirujanos del grupo con más experiencia, de más de io años, fueron quienes practicaron la mayoría $(82,7 \%)$ de las colecistectomías por laparoscopia en la institución. Cinco (o,3I \%) pacientes fueron sometidos a colecistectomía subtotal.

La duración del procedimiento mostró un amplio rango, entre 18 y 555 minutos, y su rango intercuartílico fue de 47 a 84 minutos; el tiempo más prolongado (555 minutos) correspondió a una reconstrucción biliar durante el mismo acto quirúrgico. 
Del total de pacientes, se registraron 8 muertes relacionadas con el procedimiento en los primeros 30 días, para una mortalidad global de $0,49 \%$. De los fallecidos, 5 eran mayores de 75 años y algunos tenían otras enfermedades de base, como cirrosis hepática o enfermedad cardiaca. En el estudio de histopatología de 7 $(0,43 \%)$ pacientes, se reportó cáncer de vesícula.

\section{Lesiones de la vía biliar}

La incidencia de lesión de las vías biliares fue de $0,87 \%$ (I4 pacientes). Los factores de riesgo asociados con una colecistectomía difícil, y que pudieran tener alguna relación con la lesión de las vías biliares, como diabetes mellitus, cirugía previa, cirrosis o edad mayor a 65 años 5, fueron todos estudiados, pero no se encontró ninguna relación con la presentación de lesión de las vías biliares. Tampoco se encontró asociación con el tiempo de colelitiasis $(\mathrm{p}=0,18) \mathrm{ni}$ la presencia de inflamación aguda $(\mathrm{p}=0,428)$ (tabla 2).

Los factores asociados con lesión de las vías biliares fueron el sangrado operatorio $(\mathrm{p}=\mathrm{o}, 000)$ y el tiempo quirúrgico prolongado $(\mathrm{p}=\mathrm{0}, \mathrm{0oo})$. $\mathrm{La}$ estancia hospitalaria fue mayor (I Vs. I-I8 días) en los pacientes con lesión de las vías biliares $(\mathrm{p}=\mathrm{o}, \mathrm{OOI})$.

Tabla 2. Análisis bivariado de los factores de riesgo para LVB

\begin{tabular}{|c|c|c|c|c|}
\hline \multirow[t]{2}{*}{ Variable } & \multirow{2}{*}{$\begin{array}{c}\text { Total } \\
\text { (porcentaje/rango) }\end{array}$} & \multicolumn{2}{|c|}{$\begin{array}{l}\text { Lesión de vía biliar } \\
\text { (porcentaje/rango) }\end{array}$} & \multirow[t]{2}{*}{ Valor $p$} \\
\hline & & No & Si & \\
\hline Género masculino & $376(23.4 \%)$ & $373(23.2 \%)$ & $3(0.1 \%)$ & 0.999 \\
\hline Edad promedio* años ( $n=1598)$ & $46(15-92)$ & $46(15-92)$ & $48(19-69)$ & 0.783 \\
\hline Diabetes & $136(8.5 \%)$ & $134(8.4 \%)$ & $2(14.3 \%)$ & 0.337 \\
\hline Obesidad & $87(6.8 \%)$ & $85(6.7 \%)$ & $2(20.0 \%)$ & 0.142 \\
\hline Cirugía previa & $492(30.7 \%)$ & $489(30.8 \%)$ & $3(21.4 \%)$ & 0.570 \\
\hline Cirrosis & $6(0.4 \%)$ & $6(0.4 \%)$ & 0 & 0.999 \\
\hline Cirugía urgente & $1437(89.8 \%)$ & $1425(89.8 \%)$ & $12(85.7 \%)$ & 0.647 \\
\hline Colecistitis aguda $>7$ días de evolución & $69(4.8 \%)$ & $68(4.8 \%)$ & $1(7.7 \%)$ & 0.428 \\
\hline Colecistectomía total & $1595(99.6 \%)$ & $1581(99.6 \%)$ & $14(100 \%)$ & 0.999 \\
\hline Colecistectomía subtotal & $5(0.3 \%)$ & $5(0.3 \%)$ & 0 & 0.999 \\
\hline Visión crítica $(n=1600)$ & $828(51.8 \%)$ & $822(51.8 \%)$ & $6(42.9 \%)$ & 0.596 \\
\hline Disección fundocística $(n=1554)$ & $29(1.9 \%)$ & $28(1.8 \%)$ & $1(8.3 \%)$ & 0.203 \\
\hline Sangrado promedio* mL ( $n=1595)$ & $15(1-1100)$ & $39(1-1100)$ & $136(5-500)$ & 0.000 \\
\hline Lesión vascular ( $n=1473)$ & $2(0.1 \%)$ & $2(0.1 \%)$ & 0 & 0.999 \\
\hline Tiempo quirúrgico promedio* minutos & $68(18-555)$ & $61(47-83)$ & $144(40-555)$ & 0.000 \\
\hline Experiencia del cirujano $<5$ años & $34(2.1 \%)$ & $34(2.1 \%)$ & 0 & 0.999 \\
\hline
\end{tabular}

LVB: Lesión vía biliar

"Variables cuantitativas: Test de Mann-Whitney

**Variables cualitativas: Test Fisher o Chi2 


\section{Características de los pacientes con lesión de} la vía biliar

Del grupo de pacientes que tuvieron lesiones de las vías biliares, dos ingresaron para colecistectomía electiva y el resto pertenecía al grupo de colecistectomía urgente. De los sometidos a colecistectomía laparoscópica electiva, uno presentaba, como variante anatómica, un conducto cístico de implantación muy baja (casi duodenal) y requirió reconstrucción de la vía biliar. En los pacientes con lesión de las vías biliares, la mayoría de los procedimientos $(64,2 \%)$ se llevaron a cabo en las primeras 72 horas después del ingreso a la institución y se reportó visión crítica de seguridad en $6(42,9 \%)$ de ellos. La tasa de conversión a cirugía abierta fue de $2 \mathrm{I}, 4 \%{ }^{3}$ y solo se practicó colangiografía intraoperatoria en 2 (I4,29\%).

En el $85,7 \%$ de los procedimientos, asistieron cirujanos con más de Io años de experiencia, sin que se encontrara relación con la incidencia de lesión de las vías biliares. La duración del procedimiento tuvo una mediana de 70 a 140 minutos $y$, en uno de ellos, en quien se reconstruyó la vía biliar en la misma intervención quirúrgica, la cirugía tuvo una duración total de 555 minutos. El sangrado fue de más de $100 \mathrm{ml}$ en el 42,8\%. Hubo apoyo de un segundo cirujano en solo uno de los casos. En un paciente, se hizo diagnóstico intraoperatorio de lesión de las vías biliares, confirmado mediante colangiografía intraoperatoria. En siete de los I4 pacientes con lesión de la vía biliar, el diagnóstico se hizo después del egreso $y$, en el resto, durante la estancia hospitalaria.

En la mayoría de los pacientes el diagnóstico y el manejo de la lesión de las vías biliares se hicieron mediante colangiopancreatografía retrógrada endoscópica (CPRE), con papilotomía, en el 92,8 \% ${ }^{12} \mathrm{y}$, con endoprótesis (stent), en el 7I,4\%. Las características de las lesiones y su tratamiento se describen en las tablas 3 y 4 . Hubo dos lesiones complejas con sección completa del colédoco, en las cuales fue necesario reconstruir la vía biliar con hepático-yeyunostomía. Estas intervenciones fueron practicadas por un cirujano hepatobiliar, una de ellas durante el mismo acto quirurgo y la otra después de 40 días de hospi- talización, una vez lograda una mejor condición clínica.

\section{Discusión}

El advenimiento de la colecistectomía laparoscópica como el método de referencia en el tratamiento de la mayoría de afecciones de la vesícula biliar, por sus ventajas en estancia hospitalaria, dolor posoperatorio y mejores resultados cosméticos con respecto a la colecistectomía abierta, tuvo como resultado un aumento del número de lesiones de la vía biliar ${ }^{7-9}$. La incidencia en

Tabla 3. Clasificación de la lesión de la vía biliar según Strasberg ${ }^{7}$ y su tratamiento $(n=14)$

\begin{tabular}{|c|c|c|}
\hline Variable & $\mathbf{n}$ & $\%$ \\
\hline \multicolumn{3}{|l|}{ Tipo de lesión } \\
\hline $\begin{array}{l}\text { A (Fístula del cístico o pequeños } \\
\text { conductos del lecho hepático) }\end{array}$ & 9 & 64,29 \\
\hline $\begin{array}{l}\text { B (Oclusión del conducto hepático } \\
\text { derecho aberrante) }\end{array}$ & 0 & 0 \\
\hline $\begin{array}{l}\text { C (Transección del conducto hepático } \\
\text { derecho aberrante) }\end{array}$ & 0 & 0 \\
\hline $\begin{array}{l}\text { D (Daño lateral del conducto hepático } \\
\text { común (<50\% de la circunferencia) }\end{array}$ & 1 & 7,14 \\
\hline $\begin{array}{l}\text { E1 (Estenosis del conducto hepático } \\
\text { común con muñón }>2 \mathrm{~cm} \text { ) }\end{array}$ & 1 & 7,14 \\
\hline $\begin{array}{l}\text { E2 (Estenosis del conducto hepático } \\
\text { común con muñón }<2 \mathrm{~cm} \text { ) }\end{array}$ & 1 & 7,14 \\
\hline $\begin{array}{l}\text { E3 (lesión hiliar con preservación de la } \\
\text { confluencia) }\end{array}$ & 2 & 14,29 \\
\hline $\begin{array}{l}\text { E4 (Lesión hiliar con compromiso de la } \\
\text { confluencia y pérdida de la comunicación } \\
\text { de ducto hepático derecho e izquierdo) }\end{array}$ & 0 & 0 \\
\hline $\begin{array}{l}\text { E5 (Lesión del conducto hepático derecho } \\
\text { aberrante más lesión hiliar) }\end{array}$ & 0 & 0 \\
\hline \multicolumn{3}{|l|}{ Diagnóstico } \\
\hline Diagnóstico intraoperatorio & 1 & 7,14 \\
\hline Diagnóstico intrahospitalario & 6 & 42,86 \\
\hline Diagnóstico después del egreso & 7 & 50 \\
\hline Resonancia magnética & 5 & 35,71 \\
\hline \multicolumn{3}{|l|}{ Tratamiento } \\
\hline $\begin{array}{l}\text { Colangiopancreatografía retrógrada en- } \\
\text { doscópica más endoprótesis }\end{array}$ & 10 & 71,43 \\
\hline Reconstrucción de la vía biliar & 2 & 14,29 \\
\hline
\end{tabular}


Tabla 4. Características de los pacientes con lesión de la vía biliar y su manejo

\begin{tabular}{|c|c|c|c|c|c|c|c|c|c|c|c|c|c|c|}
\hline Caso & Género & Edad & $\begin{array}{l}\text { Tipo de } \\
\text { cirugía }\end{array}$ & $\begin{array}{l}\text { Tiempo de } \\
\text { colecistitis } \\
\text { (días) }\end{array}$ & $\begin{array}{c}\text { Sangrado } \\
\text { (ml) }\end{array}$ & $\begin{array}{l}\text { Tiempo } \\
\text { quirúrgico } \\
\text { (min) }\end{array}$ & Conversión & Patología & $\begin{array}{c}\text { Diagnóstico } \\
\text { intraoperatorio }\end{array}$ & $\begin{array}{c}\text { Estancia } \\
\text { hospitalaria } \\
\text { (dias) }\end{array}$ & $\begin{array}{l}\text { Tipo de } \\
\text { Lesión }\end{array}$ & Manejo & RMN & Mortalidad \\
\hline 1 & $\mathrm{H}$ & 31 & Urgente & 3 & 150 & 98 & No & IA & No & 8 & A & CPRE + PA + Stent & $\mathrm{Si}$ & No \\
\hline 2 & M & 50 & Urgente & 7 & 200 & 119 & No & $\begin{array}{l}\text { No } \\
\text { reporte }\end{array}$ & No & 1 & A & CPRE + PA + Stent & No & No \\
\hline 3 & M & 19 & Urgente & 1 & 20 & 40 & No & $\begin{array}{l}\text { No } \\
\text { reporte }\end{array}$ & No & 4 & A & CPRE + PA + Stent & No & No \\
\hline 4 & M & 64 & Urgente & 4 & 100 & 54 & No & $\begin{array}{l}\text { No } \\
\text { reporte }\end{array}$ & No & 47 & A & $\mathrm{LPL}+\mathrm{CPRE}+\mathrm{PA}+$ Stent & No & No \\
\hline 5 & M & 30 & Urgente & 0 & 5 & 65 & No & $\begin{array}{l}\text { No } \\
\text { reporte }\end{array}$ & No & 1 & A & CPRE + PA & $\mathrm{Si}$ & No \\
\hline 6 & M & 65 & Urgente & 1 & 50 & 56 & No & $\begin{array}{l}\text { No } \\
\text { reporte }\end{array}$ & No & 1 & E3 & $\mathrm{LPL}+\mathrm{CPRE}+\mathrm{PA}+$ Stent & No & No \\
\hline 7 & $\mathrm{M}$ & 52 & Urgente & - & 100 & 99 & No & $\begin{array}{l}\text { No } \\
\text { reporte }\end{array}$ & No & 70 & E2 & Hepaticoyeyunostomía & $\mathrm{Si}$ & No \\
\hline 8 & M & 67 & Urgente & 8 & 200 & 98 & No & $\begin{array}{l}\text { No } \\
\text { reporte }\end{array}$ & No & 3 & A & CPRE + PA + Stent & $\mathrm{Si}$ & No \\
\hline 9 & M & 69 & Electiva & 0 & 200 & 120 & No & $\begin{array}{l}\text { No } \\
\text { reporte }\end{array}$ & No & 1 & A & CPRE + PA + Stent & No & No \\
\hline 10 & M & 40 & Electiva & 0 & 80 & 555 & $\mathrm{Si}$ & $\begin{array}{l}\text { No } \\
\text { reporte }\end{array}$ & SI & 5 & E1 & Hepaticoyeyunostomía & No & No \\
\hline 11 & $\mathrm{H}$ & 23 & Urgente & 5 & 50 & 70 & No & $\begin{array}{l}\text { No } \\
\text { reporte }\end{array}$ & No & 1 & E3 & CPRE + PA + Stent & No & No \\
\hline 12 & M & 30 & Urgente & 2 & 50 & 190 & $\mathrm{Si}$ & IC & No & 20 & A & CPRE + PA + Stent & $\mathrm{Si}$ & No \\
\hline 13 & M & 51 & Urgente & 1 & 200 & 140 & No & $I A+I C$ & No & 2 & D & CPRE + PA + Stent & No & No \\
\hline 14 & $\mathrm{H}$ & 46 & Urgente & 0 & 500 & 275 & $\mathrm{Si}$ & $\begin{array}{l}\text { No } \\
\text { reporte }\end{array}$ & No & 56 & A & CPRE + PA + Stent & No & No \\
\hline
\end{tabular}

CPRE: IA: inflamación aguda; IC: inflamación crónica; colangiopancreatografía retrógrada endoscópica; PA: papilotomía; LPL: lavado peritoneal por laparoscopia; LPA: lavado peritoneal abierto.

casos sometidos colecistectomía laparoscópica se estima entre 0,3 y I,5 \% ${ }^{8,10-12}$ a nivel mundial, y es mayor $(0,72 \%)$ cuando se utiliza la técnica de un solo puerto, en comparación con o, I a 0,25 \% en la colecistectomía abierta.

Actualmente, existen distintas clasificaciones de las lesiones de la vía biliar (Strasberg, Bismuth, Stewart-Way, Hanover, Neuhaus) para su caracterización y tratamiento ${ }^{7, \mathrm{H}, \mathrm{r} 2}$. Estas lesiones no pocas veces se acompañan de lesiones vasculares ${ }^{12-17}$. De todos estos sistemas de clasificación, los más utilizados son los propuestos por Strasberg y por Bismuth. En el presente estudio, la mayoría de las lesiones fueron de tipo A.
Debido a que la premisa más importante en el manejo de la lesión biliar iatrogénica es su prevención, la Society of American Gastrointestinal and Endoscopic Surgeons (SAGES) publicó las seis estrategias para adoptar una cultura universal de seguridad en la colecistectomía laparoscópica, con el objetivo de minimizar el riesgo de lesión de la vía biliar ${ }^{18}$. Dichas estrategias son las siguientes.

I. Uso del método de la visión crítica de seguridad: esclarecer el triángulo hepatocístico, separando todo el tejido graso y fibroso, separar el tercio inferior de la vesícula biliar de la placa cística, y verificar que haya solo 
dos estructuras entrando y saliendo de la vesícula.

2. Hacer una pausa intraoperatoria antes de colocar los clips y de cortar o seccionar cualquier estructura tubular.

3. Entender las potenciales variantes anatómicas.

4. Hacer uso liberal de la colangiografía u otros métodos para dibujar el árbol biliar durante la cirugía, cuando se considere necesario.

5. Reconocer cuándo la disección se acerca a una zona de gran peligro y suspenderla antes de entrar en ella. Finalizar la operación por un método distinto a la colecistectomía (colecistostomía, colecistectomía parcial, conversión a técnica abierta), si es necesario.

6. Solicitar la ayuda de otro cirujano cuando la disección o las condiciones sean difíciles.

Llama la atención que, aunque la visión crítica de seguridad introducida por Strasberg se convierte en unos de los factores más importantes para prevenir la lesión de las vías biliares durante la colecistectomía por laparoscopia ${ }^{19}$, en este estudio, solo el 5I,7 \% de los cirujanos la describió en las notas operatorias. Asimismo, en el grupo de los pacientes con lesión de las vías biliares, la visión crítica de seguridad se reportó tan solo en $42 \%$ de los casos.

Aunque este importante paso no quedara registrado en la descripción del procedimiento, no necesariamente significa que no se haya hecho, pero sí da paso al cuestionamiento sobre si la estrategia es o no aplicada de manera rutinaria en la ejecución de la técnica y, además, genera un margen de duda en el hipotético caso de alguna demanda médico-legal. Se debe tener en cuenta que, en Estados Unidos, algunos expertos en litigios por lesión de la vía biliar (médicos y no médicos) consideran a la lesión que ocurre por identificación errónea de la vía biliar como un acto de negligencia al tener la posibilidad de conversión a cirugía abierta, la de colangiografía intraoperatoria o la de terminar el procedimiento, entre otras alternativas (colecistectomía subtotal, colecistostomía, etc. ${ }^{6}$.
Debido a estas nefastas consecuencias legales, consideramos importante que se describa siempre si se obtuvo o no una visión crítica de seguridad en la nota operatoria y, cuando no sea posible, sustentarlo con los argumentos necesarios. La colangiografía intraoperatoria, que puede ser útil en casos seleccionados para prevenir la lesión de la vía biliar ${ }^{20,21}$, se utilizó solo en 7 $(0,44 \%)$ pacientes.

Las lesiones de las vías biliares pueden derivar en complicaciones mayores, como colangitis, cirrosis biliar, hipertensión portal y muerte, y su manejo puede requerir de procedimientos complejos, como una reconstrucción de la vía biliar ${ }^{22-25}$, o puede ser necesario, inclusive, un trasplante hepático ${ }^{22,26,27}$. Algunos autores, como Deziel, et al., en grandes series sobre procedimientos $(\mathrm{n}=77.604)$, realizados por $5.358 \mathrm{ciru}-$ janos en 4.292 hospitales, han reportado una tasa de $0,59 \%$ de lesiones de la vía biliar y una de $0,05 \%$ de lesiones vasculares abdominales; estas últimas son las lesiones con mayor mortalidad, si se compromete la aorta abdominal, la vena porta o el duodeno ${ }^{8}$.

En un estudio más pequeño, Keleman, et al., reportaron lesiones vasculares acompañando la lesión biliar hasta en I8 \% de los casos, las cuales son más frecuentes cuando las lesiones de la vía biliar son más complejas. Dicho estudio establece la incidencia de compromiso vascular de acuerdo con el tipo de lesión: de tipo B, $2 \%$; de tipo C, o \%; de tipo D, $23 \%$; de tipo EI, $19 \%$; de tipo E2, $30 \%$; de tipo E3, $9 \%$; de tipo E4, I2 \%, y de tipo $\mathrm{E}_{5}, 5 \%{ }^{13}$. En $56 \%$ de los casos, la lesión fue reconocida durante el procedimiento quirúrgico, alcanzándose una mortalidad del $33 \%$.

En el presente estudio, la incidencia de lesión de la vía biliar fue de $0,87 \%$, un valor acorde con lo reportado y no hubo lesión vascular asociada. Solo una lesión de las vías biliares fue reconocida durante el intraoperatorio. La mortalidad global fue de $0,49 \%$, una cifra similar a la reportada en otras series ${ }^{28-30}$.

La edad del paciente no fue un factor de riesgo para lesión de la vía biliar, como lo reportan otros estudios ${ }^{31}$. El 64,29 \% de las lesiones co- 
rrespondió a las de tipo A de la clasificación de Strasberg, una cifra acorde con lo reportado por otros autores ${ }^{32}$, y cuyas manifestaciones muchas veces son más larvadas. Esto explica por qué, en algunos casos, el diagnóstico fue tardío e, incluso, se autorizó el egreso.

De los pacientes con lesión de las vías biliares tratados mediante CPRE, en 7I,4\% se requirió endoprótesis (stent). No obstante, llama la atención que, de los 9 pacientes con lesión de tipo A, 6 (66 \%) tenían coledocolitiasis u estenosis distal asociadas, lo cual pudo haber sido un factor asociado o causal de la fístula, por aumento de la presión dentro la vía biliar.

De las variables analizadas, el volumen del sangrado y el tiempo operatorio tuvieron relación con la lesión de las vías biliares $(\mathrm{p}=0,000$, ambos), lo cual habla de procedimientos posiblemente más difíciles, con tiempos operatorios más prolongados. No se encontró relación con factores de riesgo para lesión de las vías biliares, como duración de la colecistitis, diabetes mellitus, obesidad, cirugía previa o edad del paciente, probablemente, debido a la baja incidencia de lesiones biliares.

Se practicó colecistectomía laparoscópica en $70 \%$ de los pacientes en un tiempo de 72 horas o menos después del ingreso, lo cual se ha relacionado con menor morbilidad, menor estancia hospitalaria, menos costos y una mayor tasa de recuperación; esto puede reducir el número de colecistectomías laparoscópicas difíciles por reducción de la posibilidad de inflamación subaguda y, de forma indirecta, relacionarse con una menor incidencia de lesión de las vías biliares $5,33,34$.

La tasa de conversión a cirugía abierta fue relativamente baja (o,8 \%) en el grupo estudiado, en comparación con lo informado en la literatura científica: $4,6 \%$ electiva y $9,4 \%$, urgente, con reportes de hasta el $\mathrm{I} 5 \%{ }^{5}$, pero sí mucho mayor en casos de lesión de la vía biliar (2I,4\%).

La estancia hospitalaria fue mucho mayor en los casos de lesión de la vía biliar, lo cual se entiende por el periodo requerido para el manejo de la complicación y la recuperación del paciente. Esto contrasta con los pacientes sin lesión que se operan de forma electiva, en quienes la colecistectomía laparoscópica puede ser un procedimiento ambulatorio ${ }^{35}$.

Este estudio cuenta con las limitaciones propias de los estudios retrospectivos, como la falta de control de los los sesgos y la perdida de datos en algunas de las variables estudiadas.

\section{Conclusiones}

La lesión de vía biliar es una complicación grave de la colecistectomía laparoscópica, asociada a alta morbilidad. La mejor aproximación de manejo a esta complicación es la prevención, motivo por el cual es fundamental para el cirujano conocer y aplicar las estrategias de colecistectomía laparoscópica segura sugeridas por los expertos. Nuestro estudio mostró que la colecistectomía por laparoscopia en un centro académico como el Hospital Universitario San Vicente Fundación, es un procedimiento seguro y con una incidencia baja de lesión de la vía biliar (o,87\%), tasa similar a la reportada por otros centros de excelencia. La mayoría de las lesiones encontradas fueron leves (de tipo A en la clasificación de Strasberg) y su tratamiento con colangiopancreatografía retrógrada fue exitoso.

En este estudio, los factores de riesgo asociados con lesión de las vías biliares, fueron el mayor sangrado y el mayor tiempo operatorio. Según nuestro conocimiento, a la fecha, esta es la serie más grande de su tipo publicada en el país.

Conflictos de interés. Ninguno declarado

Fuentes de financiación. Autofinanciado

\section{Referencias}

I. Ángel A, Rosero G. Coledocolitiasis. Guías de manejo en cirugía. Bogotá: Asociación Colombiana de Cirugía. Fecha de consulta: noviembre 25 de 20I8. Disponible en: https://www.ascolcirugia.org/images/resources/ PDF/guiasCirugia/coledocolit iasis.pdf.

2. Strasberg SM. Error traps and vasculo-biliary injury in laparoscopic and open cholecystectomy. J Hepatobiliary Pancreat Surg. 2008;15:284-92.

3. Way LW, Stewart L, Gantert W, Liu K, Lee CM, Whang $\mathrm{K}$, et al. Causes and prevention of laparoscopic bile 
duct injuries: Analysis of 252 cases from a human factors and cognitive psychology perspective. Ann Surg. 2003;237:460-9.

4. Strasberg SM. Avoidance of biliary injury during laparoscopic chelocystectomy. J Hepatobiliary Pancreat Surg. 2002;9:543-7.

5. Álvarez LF, Rivera D, Esmeralda ME, García MC, Toro DF. Colecistectomía laparoscópica difícil, estrategias de manejo. Rev Colomb Cir. 2013;28:186-95.

6. Strasberg SM. Biliary injury in laparoscopic surgery: Part I. Processes used in determination of standard of care in misidentification injuries. J Am Coll Surg. 2005;201:598-603.

7. Stewart L. Iatrogenic biliary injuries: Identification, classification, and management. Surg Clin North Am. 20I4;94:297-3I0.

8. Deziel DJ, Millikan KW, Economou SG, Doolas A, Ko ST, Airan MC. Complications of laparoscopic cholecystectomy: A national survey of 4,292 hospitals and an analysis of 77,604 cases. Am J Surg. 1993;I65:9-I4.

9. Olaya C, Carrasquilla G. Meta-análisis de efectividad de la colecistectomía laparoscópica frente a la abierta. Rev Colomb Cir. 2006;21:104-I5.

Io. Sarno G, Al-Sarira AA, Ghaneh P, Fenwick SW, Malik HZ, Poston GJ. Cholecystectomy-related bile duct and vasculobiliary injuries. Br J Surg. 2012;99:II29-36.

II. Rose JB, Hawkins WG. Diagnosis and management of biliary injuries. Curr Probl Surg. 20I7;54:406-35.

I2. Bektas H, Schrem H, Winny M, Klempnauer J. Surgical treatment and outcome of iatrogenic bile duct lesions after cholecystectomy and the impact of different clinical classification systems. Br J Surg. 2007;94:III9-27.

I3. Keleman AM, Imagawa DK, Findeiss L, Hanna MH, Tan $\mathrm{VH}, \mathrm{Katz} \mathrm{MH}$, et al. Associated vascular injury in patients with bile duct injury during cholecystectomy. Am Surg. 20II;77:1330-4.

I4. Pulitan C, Parks RW, Ireland H, Wigmore SJ, Garden OJ. Impact of concomitant arterial injury on the outcome of laparoscopic bile duct injury. Am J Surg. 20II;20I:238-44.

I5. Tzovaras G, Dervenis C. Vascular injuries in laparoscopic cholecystectomy: An underestimated problem. Dig Surg. 2007;23:370-4

I6. Li J, Frilling A, Nadalin S, Paul A, Malagò M, Broelsch CE. Management of concomitant hepatic artery injury in patients with iatrogenic major bile duct injury after laparoscopic cholecystectomy. Br J Surg. 2008;95:460-5.
I7. Strasberg SM, Helton WS. An analytical review of vasculobiliary injury in laparoscopic and open cholecystectomy. HPB. 20II;I3:I-I4.

18. The SAGES Safe Cholecystectomy Program. Strategies for minimizing bile duct injuries: Adopting a universal culture of safety in cholecystectomy. Fecha de consulta: noviembre 25 de 20I8. Disponible en: http://www.sages. org/safe-cholecystectomy-program/.

19. Strasberg SM, Brunt LM. Rationale and use of the critical view of safety in laparoscopic cholecystectomy. J Am Coll Surg. 2010;2II:I32-8.

20. Ford JA, Soop M, Du J, Loveday BPT, Rodgers M. Systematic review of intraoperative cholangiography in cholecystectomy. Br J Surg. 2012;99:I60-7.

2I. Álvarez FA, De Santibañes M, Palavecino M, Sánchez $\mathrm{R}$, Mazza O, Arbues G, et al. Impact of routine intraoperative cholangiography during laparoscopic cholecystectomy on bile duct injury. Br J Surg. 20I4;IOI:677-84.

22. Strasberg SM, Gouma DJ. "Extreme" vasculobiliary injuries: Association with fundus-down cholecystectomy in severely inflamed gallbladders. HPB. 20I2;I4:I-8.

23. Jabłońska B, Lampe P, Olakowski M, Górka Z, Lekstan A, Gruszka T. Hepaticojejunostomy Vs. end-to-end biliary reconstructions in the treatment of iatrogenic bile duct injuries. J Gastrointest Surg. 2009;13:I084-93.

24. Navarrete C, Gobelet JM. Treatment of common bile duct injuries after surgery. Gastrointest Endosc Clin N Am. 2012;22:539-53.

25. Stewart L, Way LW. Bile duct injuries during laparoscopic cholecystectomy. Factors that influence the results of treatment. Arch Surg. 1995;130:II23-9.

26. Parrilla P, Robles R, Varo E, Jiménez C, Sánchez-Cabús S, Pareja E. Liver transplantation for bile duct injury after open and laparoscopic cholecystectomy. Br J Surg. 20I4;IOI:63-8.

27. De Santibañes E, Ardiles V, Gadano A, Palavecino M, Pekolj J, Ciardullo M. Liver transplantation: The last measure in the treatment of bile duct injuries. World J Surg. 2008;32:I7I4-2I.

28. Sanchez-Beorlegui J, Monsalve-Laguna E, Aspiroz-Sancho A, Moreno De Marcos N. Colecistectomía laparoscópica en octogenarios. Rev Colomb Cir. 2008;23:I63-I45.

29. Nilsson E, Fored CM, Granath F, Blomqvist P. Cholecystectomy in Sweden 1987-99: A nationwide study of mortality and preoperative admissions. Scand J Gastroenterol. 2005;40:I478-85. 
30. Shea JA, Healey MJ, Berlin JA, Clarke JR, Malet PF, Staroscik RN, et al. Mortality and complications associated with laparoscopic cholecystectomy: A meta-analysis. Ann Surg. 1996;224:609-20.

3I. Domínguez, L, Rivera A, Herrera W. Colecistectomía de urgencia por laparoscopia por colecistitis aguda en adultos mayores. Rev Colomb Cir. 20II;26:93-IOO.

32. Pitt HA, Sherman S, Johnson MS, Hollenbeck AN, Lee J, Daum MR, et al. Improved outcomes of bile duct injuries in the 2Ist century. Ann Surg. 20I3;258:490-9.

33. Yamashita Y, Takada T, Strasberg SM, Pitt HA, Gouma DJ, Garden OJ, et al. TGi3 surgical management of acute cholecystitis. J Hepatobiliary Pancreat Sci. 2013;20:89-96.

34. Menahem B, Mulliri A, Fohlen A, Guittet L, Alves A, Lubrano J. Delayed laparoscopic cholecystectomy increases the total hospital stay compared to an early laparoscopic cholecystectomy after acute cholecystitis: An updated meta-analysis of randomized controlled trials. HPB. 2015;17:857-62.

35. Acuña J, Bonfante J, Galeano N, Carrasquilla R, Manjarrez A, Alcalá R. Colecistectomía laparoscópica con manejo ambulatorio ultracorto. Rev Colomb Cir. 2015;30:II9-24. 